\title{
Image Fusion and Evaluation of Geological Disaster Based on Remote Sensing
}

\author{
http://dx.doi.org/10.3991/ijoe.v10i4.3705 \\ Juan.Wang. ${ }^{1,2}$, Huajun.Wang. ${ }^{*}$, Yinghao.Li. ${ }^{1}$ and Hairui.Chen. ${ }^{1}$ \\ ${ }^{1}$ Chengdu University of Technology, Chengdu, China \\ ${ }^{2}$ China West Normal University, Nanchong, China
}

\begin{abstract}
The investigation of geological disaster in our article locates in southern Australia, which is characterized by wide range, high relief, inaccessibility and other unfavorable factors. Multi-spectral ETM+ and SPOT 5 pan images were selected as the remote sensing data source, and Brovey transform (BT), intensity-hue-saturation (IHS), principal component analysis (PCA), high-pass filtering (HPF) and modified Gram-Schmidt (MGS) methods were used for image fusion. A comparison has been conducted between the resultant fusion images to assess the image quality both in subjective and objective evaluation. The results show that, the MGS method is the optimal image fusion method for geological disaster interpretation, and can provide abundant textural and spectral information in interpreting such geological disasters as landslide, rock fall and debris flow.
\end{abstract}

Index Terms-ETM+, geological disaster, image fusion, remote sensing, SPOT5.

\section{INTRODUCTION}

Due to wide scope and bad natural conditions, it is extremely difficult for conventional ground investigation of geological disaster to perform. Remote sensing technology can obtain surface information quickly and efficiently, so it plays an important part in investigation of geological disaster $[1,2,3]$. In order to reflect the morphological differences adequately between geological disaster and surrounding environment, high resolution images are required in geological disaster investigation. Currently, high-resolution remote sensing satellites (such as QuickBird and IKONOS) can meet the requirements of spatial resolution for survey of geological disasters, but the high cost and large volume limit the applications. The Landsat7 ETM+ images contain high spectrum information, and enjoy $15 \mathrm{~m}$ panchromatic resolution while maintaining the continuity of TM data. SPOT5 Pan has high spatial resolution, but is inferior to ETM+ as to multi-spectral information and image cost. Therefore, the fused images based on ETM+ and SPOT5 Pan can give full play to their respective advantages in terms of spatial resolution and spectral information, and have relative high performance-price ratio, which are ideal data sources for remote sensing survey of geological disasters.

Until recently, a few approaches have been widely used in high-resolution panchromatic images and lowresolution multi-spectral images fusion purpose. Methods like Brovey transform (BT) [4], intensity-hue-saturation (IHS) [5], principal component analysis (PCA) [6], highpass filtering (HPF) [7] and Gram-Schmidt methods [8] are all adopted in geological image fusion. And the acquisition of high quality spectral information is very crucial for most of the sensing applications, such as soil \& water conservation, geology and agrology. Taking ETM+ and SPOT5 satellite images in southern Australia as an example, our article analyzed and estimated the performances of above mentioned fusion methods in geological investigation.

The remainder of this article is organized as follows we introduce preprocessing on source images and fusion methods in Section 2. This is followed by a quality evaluation on resultant images both in subjective and objective ways. Section 4 presents the results of the interpretation on remote sensing images. Finally, the last Section concludes with a discussion of our work and recommends MGS method in survey of geological disasters.

\section{SOURCE IMAGES AND Fusion Methods}

\section{A. Bands Selection and Preprocessing}

Both the ETM+ and SPOT5 can provide multi-spectral images. The former has eight bands and its spectral range is from 0.45 to $12.50 \mu \mathrm{m}$ while the latter has five bands and its wavelength ranges from 0.48 to $1.75 \mu \mathrm{m}$. One of the key steps for remote sensing image fusion is band integration, the optimum band integration can take full advantage of fusion technology to achieve the best fusion image, especially for fusion models like IHS and Brovey, only a three-bands integration occur, therefore the appropriate band integration makes better fusion results.

Band integration should be guided by the following principles: in so far as SPOT5 is concerned the least spectral correlation and the largest amount of information are desired. Pearson Product-moment Correlation Coefficient, also known as PCCs, it is a measure of the strength and direction of the linear relationship between two multi-dimensional random variables [9]. The Optimum Index Factor (OIF) is a statistic value that can be used to select the optimum combination of three bands in a satellite image with which you may create a color composite. The optimum combination of bands out of all possible three-band combinations is the one with the highest amount of information. The statistics show that the red band of SPOT5 is usually adopted to perform geological interpretation, identification with crop, bare soil and rock, so red band must be selected. In this case, PCCs of bands pair NIR-R (near-infrared, red), NIRSWIR (near-infrared, short-wave infrared) and R-SWIR (red, short-wave infrared) are $0.365,0.695$ and 0.702 
respectively, which is the least value comparatively. And combination NIR-R-SIR enjoys the highest OIF, 57.368, hence this one is the best.

As for multi-spectrum ETM+ images, analytical data show that band SWIR1 (short-wave infrared) owns the largest SD (standard deviation) 46.62, followed by SWIR2 35.45, NIR (near-infrared) 30.95 and R (red) 30.82 . Therefore, band SWIR1 and SWIR2 have the largest amount of data, but their data relevance is high up to 0.991 which means too many data is overlapped. Both SWIR1 and SWIR2 are short-wave infrared bands, and locate between the water absorption bands, but SWIR2 is sensitive to rocks and minerals which can be used to distinguish rock types, analyze features of hydrothermal alteration of rocks [10]. By selecting bands combination of SWIR2-NIR-Red, SWIR1-NIR-Red, SWIR2-NIR-Blue and SWIR1-NIR-Blue from a 300 by 300 sub-image and conducting contrast experiment. Results reveal that the SWIR2-NIR-Red combination reflects color difference layer good especially for the exposed bedrock and rock debris.

IHS and Brovey transformation both produce a threeband fusion image, which correspond to combination SWIR2-NIR-Red. PCA and Gram-Schmidt transformation employ SWIR2-SWIR1-NIR, Red-Green-Blue combination, which achieves a six-band contained result. In order to facilitate comparison of fusion images, PCA and Gram-Schmidt transformation often choose band combination that is corresponding to SWIR2-NIR-Red before fusion start. And the combination inherits spectral characteristics of the combo SWIR2-NIR-Red, and can achieve the best fusion effect.

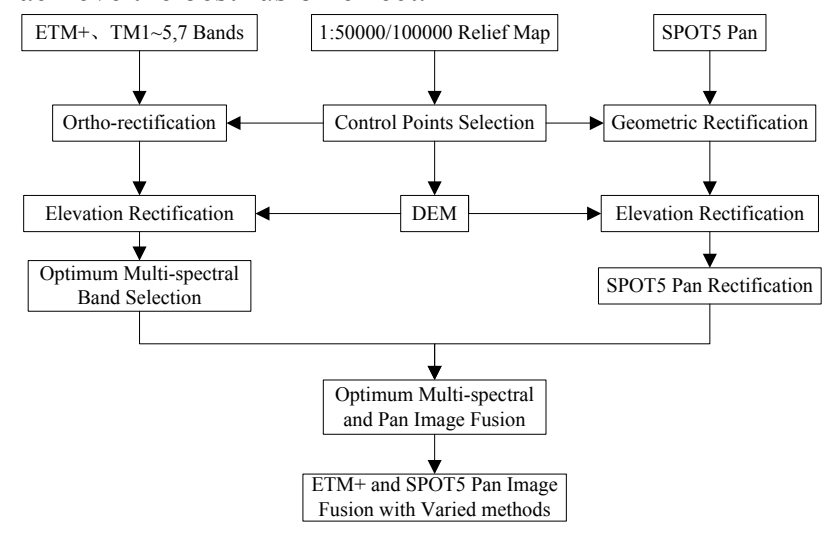

Figure 1. Flow diagram of the pretreatment process.

Pretreatments on ETM+ and SPOT5 Pan images are mainly include geometric rectification and optimum bands selection, etc., the process is shown in Figure 1.

\section{B. Fusion Methods}

There is large gap in spatial resolution between SPOT5 Pan and ETM+ multi-spectral images, we need first to ensure that registration accuracy is less than 0.5 pixel and the ETM+ is resampled to the pixel size of SPOT5 Pan by using cubic convolution for example. As we take the professional background of the remote sensing image processing staffs for geological hazard investigation into consideration, several such commonly used fusion algorithm as BT, IHS, PCA, HPF and GS are adopted for integration of the ETM+ multi-spectral and SPOT5 Pan, which are easy to implement.
(1) BT method. Brovey image fusion is also known as color normalized fusion, the idea of which is to calculate by components of color and intensity decomposed from image space of multi-spectral image [11]. This method is mainly used in spectral response range of high-resolution panchromatic image is the same or similar with that of the low-resolution multi-spectral image. The formula is:

$$
\left\{\begin{array}{l}
R_{F}=\frac{R_{M S}}{R_{M S}+G_{M S}+B_{M S}} * I_{P} \\
G_{F}=\frac{G_{M S}}{R_{M S}+G_{M S}+B_{M S}} * I_{P} \\
B_{F}=\frac{R_{M S}}{R_{M S}+G_{M S}+B_{M S}} * I_{P}
\end{array}\right.
$$

where $I_{P}$ is the source high-resolution panchromatic image, $R_{M S}, G_{M S}$ and $B_{M S}$ are the red, green and blue channel images of the source low-resolution multi-spectral images respectively; $R_{F}, G_{F}$ and $B_{F}$ are red, green and blue channel images of the fused image respectively.

The advantages of this method are the simplification of the conversion coefficients and the preservation of the spectral information from original multi-spectral image when enhancing it. The multi-spectral image must be a three-band contained true color or pseudo-color image. The method is applicable to fuse SPOT panchromatic image and related multi-spectral one, or SPOT panchromatic image and TM multi-spectral image with similar bands.

(2) IHS method. The process of RGB (red-green-blue) space or IHS (intensity-hue-saturation) space is transformed into each other is called IHS or inverse IHS transformation. In all color conversion technologies, IHS transform is the one that is most consistent with human visual system $[12,13]$. The component I are separated from $\mathrm{H}$ and $\mathrm{S}$ effectively, but the correlation between these three becomes low, the three are considered substantially independent, which is conducive to spectral information maintenance. The IHS transformation has different forms: sphere transform, cylinder transform, triangle transform and pyramid transform, the forward transformation we take is like formulas (2) and (3) show, and the inverse transformation is displayed by formulas (4).

$$
\begin{aligned}
& {\left[\begin{array}{l}
I \\
V_{1} \\
V_{2}
\end{array}\right]=\left[\begin{array}{ccc}
\frac{1}{\sqrt{3}} & \frac{1}{\sqrt{3}} & \frac{1}{\sqrt{3}} \\
\frac{1}{\sqrt{6}} & \frac{1}{\sqrt{6}} & -\frac{2}{\sqrt{6}} \\
\frac{1}{\sqrt{2}} & -\frac{1}{\sqrt{2}} & 0
\end{array}\right]\left[\begin{array}{l}
R \\
G \\
B
\end{array}\right]} \\
& H=\tan ^{-1}\left[V_{1} / V_{2}\right], S=\sqrt{V_{1}^{2}+V_{2}^{2}} \\
& {\left[\begin{array}{l}
R \\
G \\
B
\end{array}\right]=\left[\begin{array}{ccc}
\frac{1}{\sqrt{3}} & \frac{1}{\sqrt{6}} & \frac{1}{\sqrt{2}} \\
\frac{1}{\sqrt{3}} & \frac{1}{\sqrt{6}} & -\frac{1}{\sqrt{2}} \\
\frac{1}{\sqrt{3}} & -\frac{2}{\sqrt{6}} & 0
\end{array}\right]\left[\begin{array}{l}
I \\
V_{1} \\
V_{2}
\end{array}\right]}
\end{aligned}
$$


When fusing high-resolution panchromatic image and multi-spectral image using IHS transformation, you need first transform the low-resolution multi-spectral image into IHS space and get I, H and $\mathrm{S}$ components. Then conduct contrast stretching to the high-resolution image to make it have the same mean and variance with component I. And replace the component I with the stretched highresolution image, with which the $\mathrm{H}$ and $\mathrm{S}$ components are transformed inversely together. Thus the obtained image not only has high spatial resolution, but also enjoys the same hue and saturation with the original image.

(3) PCA transform method. Principal component analysis (PCA) is a multi-dimensional (multi-band) orthogonal linear transformation on the basis of the statistical characteristic [14], because the most commonly known mathematical transformation is called K-L (Karhunen-Loeve) transformation, so the PCA transformation is often named K-L transformation.

A standard transformation matrix is needed before the PCA transformation can start when dealing with remote sensing image data. And convert original image data into a new group -- principal component data, thereby extracting the principal component features, all new features constructed by this method are all linear functions with respect to the original feature. The transformation formula can be expressed as:

$$
Y=T X
$$

where $X$ is the data matrix of the original image before transformation. And $Y$ is the data matrix after transformation. $T$ is the transformation matrix. Generally, each row vector of the converted data matrix is called one of the principal components of K-L transformation. According to the definition of the K-L transformation, multi-spectral image conversion process can be summarized below:

i) The multi-spectral image data matrix is $X$ (each row of the matrix represents an image of certain band), assuming multi-spectral image is composed of $m$ images in different bands, there are $n$ pixels in each image. The data matrix $X$ and covariance matrix $C$ can be depicted as formulas (6) and (7) respectively:

$$
\begin{aligned}
X & =\left[\begin{array}{ccccc}
x_{11} & \mathrm{~L} & x_{1 j} & \mathrm{~L} & x_{1 n} \\
\mathrm{M} & \mathrm{M} & \mathrm{M} & \mathrm{M} & \mathrm{M} \\
x_{i 1} & \mathrm{~L} & x_{i j} & \mathrm{~L} & x_{i n} \\
\mathrm{M} & \mathrm{M} & \mathrm{M} & \mathrm{M} & \mathrm{M} \\
x_{m 1} & \mathrm{~L} & x_{m j} & \mathrm{~L} & x_{m n}
\end{array}\right] \\
C & =\left[\begin{array}{ccccc}
\sigma_{11}^{2} & \mathrm{~L} & \sigma_{1 i}^{2} & \mathrm{~L} & \sigma_{1 m}^{2} \\
\mathrm{M} & \mathrm{M} & \mathrm{M} & \mathrm{M} & \mathrm{M} \\
\sigma_{i 1}^{2} & \mathrm{~L} & \sigma_{i i}^{2} & \mathrm{~L} & \sigma_{i m}^{2} \\
\mathrm{M} & \mathrm{M} & \mathrm{M} & \mathrm{M} & \mathrm{M} \\
x_{m 1} & \mathrm{~L} & x_{m i} & \mathrm{~L} & x_{m m}
\end{array}\right]
\end{aligned}
$$

ii) Calculate eigenvalue $\lambda$ and eigenvector $U$ of covariance matrix $C$, with which the transformation matrix $T$ is composed, if we form matrix $U$ with eigenvectors column by column then the transpose matrix of $U$ is the coefficient matrix $T$ of K-L transformation.

iii) Obtain new matrix $Y$ by using specific expression for K-L transformation $Y=U^{T} X=T X$. Each row vector
$Y_{i}$ of matrix $Y$ corresponds to one principal component, in which $Y_{I}$ is called the first principal component and contains the maximum amount of information while the information of other principal components decrease gradually. The pixel numbers of each principal component are all $n$, which is the same as that of a single band image. The covariance between any two principal components is zero, which ensures that there is no duplication and redundancy of information among principal components.

As for K-L transformation, compress or integrate multiband image information into one image which can also be regarded as the way of the image fusion. Hence, for this type of image fusion, each band contributes to the fusion result to the most. Due to the cumulative contribution rate of the first three principal components can reach up to $98 \%$, so the original band information are concentrated in the first three principal components image while the rest of the images composed by other principal components are basically noise, in which a dimension reduction process is implemented. If assign the first three principal component images to red, green and blue channels respectively, as a result the purpose of image fusion using $\mathrm{K}-\mathrm{L}$ transformation is achieved.

This method is effective when fusing TM and SPOT data, or fusing SPOT multi-spectral and panchromatic data. The fused image inherits the high spatial resolution and high spectral resolution characteristics of the original two images, and retains high frequency information of the original image. Objects on target image enjoy detailed features clearer and have spectral information richer [15].

(4) HPF method. High-pass filtering fusion method is a way that less spectral information lost. By first using a small space HPF filter the high-resolution image, as the filtered image not only retains high frequency components related to spatial information but also drops most of the spectral information, so the HPF filtered result is added to spectral images. By doing so, the spatial information from high-resolution image and spectral information from multi-spectral image are integrated, and the fused image is characterized by the highlighted high-frequency fused image. Either single or multiple band images can be fused in this way [6], the mathematical model is as follows:

$$
B_{i F}=B_{i}+H P(A)
$$

where $B_{i}$ denotes low-resolution multi-spectral image, $B_{i F}$ denotes the fused multi-spectral image, $A$ is highresolution panchromatic image and $H P(A)$ represents the obtained high frequency image by conducting spatial HPF on $A$.

HPF method is simple to use and is not limited by the number of bands of multi-spectral images. Only small part of spectral distortion occurs by taking this method, but the spatial resolution is improved relatively small.

(5) The modified Gram-Schmidt (MGS) method. Compared with PCA transformation components produced by MGS are just orthogonal and are not significantly different, while the first component does not changed before and after the conversion. The specific steps of MGS transformation is shown as Figure 2:

i) Simulate low-resolution panchromatic image. According to the prior knowledge, we need first simulate the first component of orthogonal vector and then work out the other orthogonal vectors for MGS transformation in remote sensing MGS image fusion. The quality of the 
PAPER

IMAGE Fusion AND Evaluation of GEOLOGICAl DisASTER BASED ON REMOTE SENSING

first simulated component can greatly influence the final fused image, which is the key of the MGS fusion method.

ii) The simulated image is regarded as the first component and performs calculation in MGS transformation.

iii) Adjust high-resolution image and make it match the first simulated component, with which the first component can be replaced. The first component and other components are organized to obtain the fused image by conducting inverse MGS transformation.

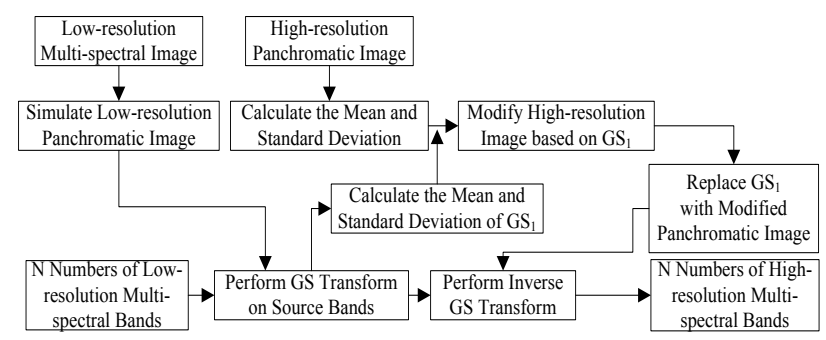

Figure 2. Fusion method of modified Gram-Schmidt transformation.

\section{IMAGE FUSION EVALUATION CRITERIA}

Quality and effect evaluation of the fused image is an important and complex work in image fusion, which has not been solved for many years, since it is hard to give the perfect evaluation criteria. The main reasons lie in several aspects: on one hand, in most cases we can not get the standard fusion image in advance, the evaluator has no reference object. On the other hand, for the specific fusion method different source image can produce different fusion effect, for the specific fusion algorithm and source image as the viewer may interests in different part of the image, the evaluation effect is different too. Different application purposes have different requirements on source images, which lead to the selected fusion method varied. Hence, many scholars are committed to find subjective, objective and quantitative evaluation methods, and hope to make subjective, objective and quantitative evaluation indicators and guidelines when using a variety of fusion methods, fusion rules and fusion operator.

At present, there are two main image quality and effect evaluation approaches, namely, the subjective and the objective evaluation.

(1) The visual assessment (subjective evaluation) is the processes that evaluate the quality of the images with the naked eyes on the basis of subjective feeling and statistical results. Subjective assessment method has advantages of simple and intuitive, and apparent image information can be interpreted fast and convenient.

Figure 3(a)-(g) are the source and fused images. As for spectral information, all the fused images are enhanced in different degree, the worst one is IHS method Figure 3(d) while PCA Figure 3(e) and MGS Figure 3(g) methods are in the first grade which have little distortion in spectrum. As far as the image contents are concerned, all the fused images have richer spatial information than original multispectral image Figure 3(a) in that an obvious sea water area can be seen in Figure 3(a) and shrink to the same size as panchromatic Figure 3(b) shows in all fused images. As IHS Figure 3(d) and HPF Figure 3(f) show, they have relatively low texture when compared to other fused image. And PCA Figure 3(e) enjoys clearer texture information especially for the field and wasteland, but the spectrum distortion is distinct and is different from other images, for example, the color of sea water area in PCA Figure 3(e) is changed. As we can see that the MGS method Figure $3(\mathrm{~g})$ has the best visual effect such as definition, color fidelity, contrast and color hierarchy, and the overall effect is better than that of other images.

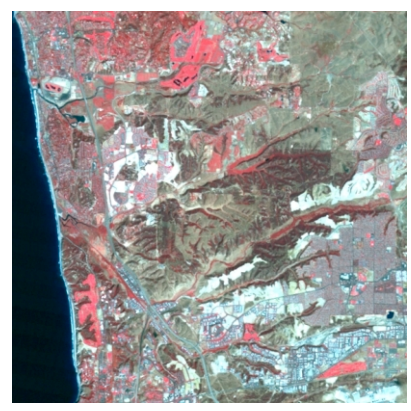

(a)

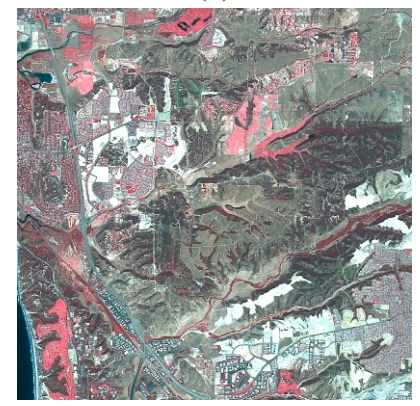

(c)

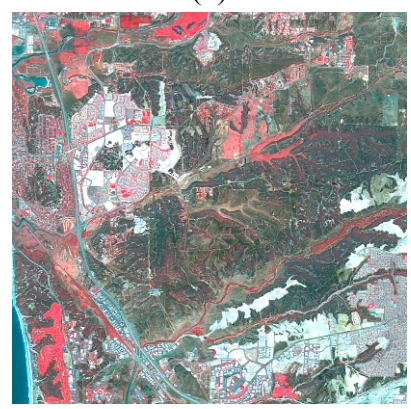

(e)

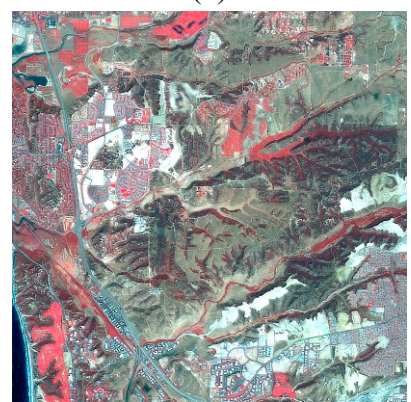

(g)

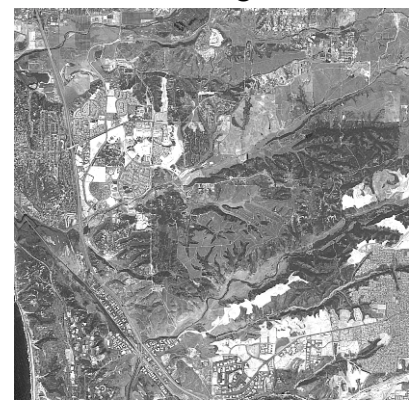

(b)

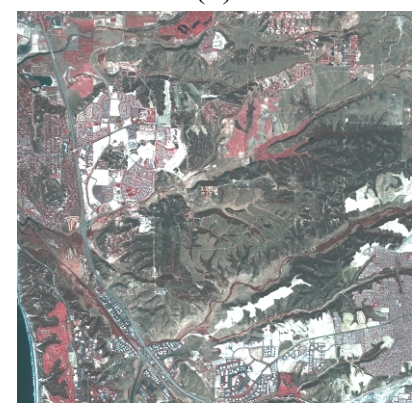

(d)

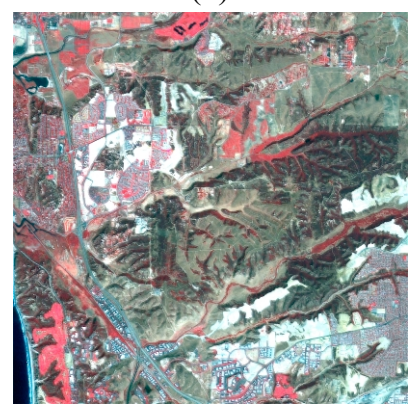

(f)
Figure 3. The source and the fused images of all fusion method. (a) Multi-spectral image; (b) Panchromatic image; (c) BT fused image; (d) IHS fused image; (e) PCA fused image; (f) HPF fused image; (g) MGS fused image.

From the enlarged sub-images of Figure 4 (a) to Figure 4 (e) we label the landslide spot with white arrow, all the landslide point can be found from corresponding image. In terms of color, partially color shift and spectral degradation can be found in bank of the sea water for BT 
Figure 4(a) and IHS Figure 4(b) images. In the aspects of texture, IHS transformation has abundant texture information but locally highlight spotted phenomenon. The BT transformation image is blurry relatively, which is not conducive to interpret geological disasters. PCA Figure 4(c) and MGS Figure 4(e) fused images have high spectral fidelity, clear color levels, distinct micro-structure and MGS has the best overall effect which is conducive to visual interpretation of geological disasters.

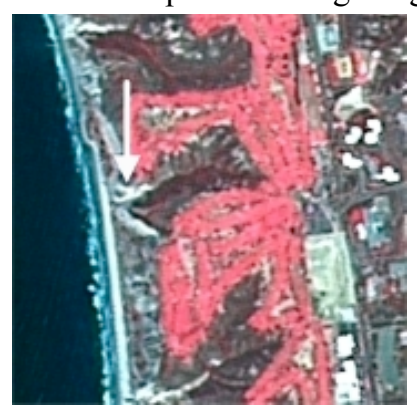

(a)

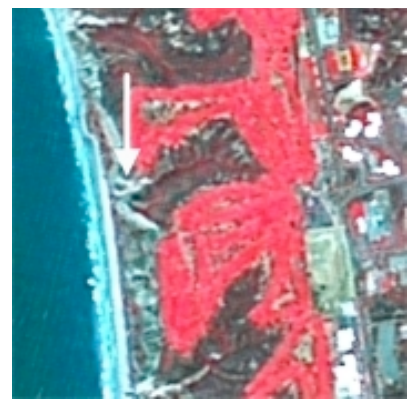

(c)

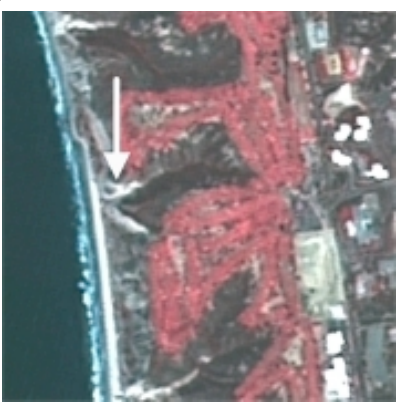

(b)

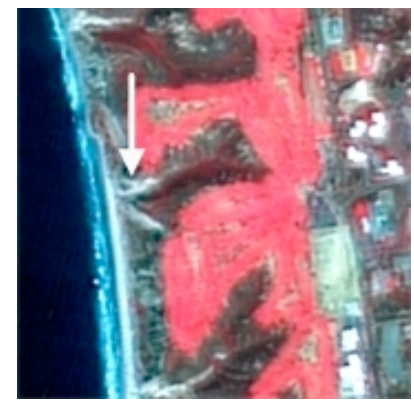

(d)

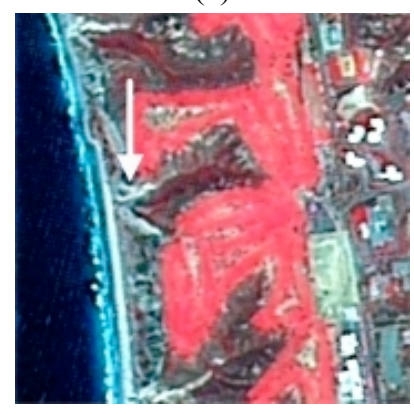

(e)

Figure 4. The comparison of landslide for different fusion images. (a) Landslide of BT fused image; (b) Landslide of IHS fused image; (c) Landslide of PCA fused image; (d) Landslide of HPF fused image; (e) Landslide of MGS fused image.

(2) When selecting the image fusion evaluation indices (mainly refers to the objective evaluation index), one can select evaluation indices according to the specific fusion purpose, the main evaluation parameters can be classified into such following classes as: spatial resolution promotion, information content, definition, spectral property and noise reduction. And we adopt some of the parameters so as to make objective evaluation for the fused image.

i) Information Entropy (IE). IE is one of the most important indicator, whose value can directly reflects the amount of information in the image. The bigger the IE is the more information is contained in a fused image.

ii) Average Gradient (AG). AG reflects the ability to express the tiny detailed contrast and texture variations in an image, and also the definition of the image which will be better with an increasing AG value.

iii) Pearson Correlation Coefficient (PCCs). PCCs reflects the correlation degree between two images, the PCCs value for a pair of source multi-spectral image and the fused one indicates the extent that the multi-spectral information that has changed. The higher the PCCs, the less the changing of spectrum will be.

iv) Image histogram is the function of gray level which represents the number of pixel that with the specific gray level, and it can reflect the frequency of certain gray level appeared. It is the probability distribution of all the gray levels.

From Table I we can see that, the entropy for all the fused images are enhanced in band1, band 2 and band 4 . HPF and MGS are superior to other methods as for band2 and band4, but in band1 IHS take the first place.

As revealed in Table II, the definition, texture and detailed contrast of all methods are enriched, which means the fused image have more spatial information than the multi-spectral image. BT and MGS methods are superior to other methods in all bands.

As can be seen in Table III, the BT and MGS methods overcome other approaches in PCCs index, which followed by IHS, HPF and PCA sequentially. We note that larger PCCs means more spectral information is maintained by the corresponding fused image.

TABLE I.

INFORMATION ENTROPY FOR ALL METHODS

\begin{tabular}{llll}
\hline & Band 1 & Band 2 & Band 4 \\
\hline Multi-spectral image & 3.0925 & 3.1318 & 3.1553 \\
BT & 3.1916 & 3.3670 & 3.3938 \\
IHS & 3.3473 & 3.1673 & 3.2126 \\
PCA & 3.2020 & 3.3404 & 3.3015 \\
HPF & 3.1033 & 3.4234 & 3.4225 \\
MGS & 3.1711 & 3.4308 & 3.4115 \\
\hline
\end{tabular}

TABLE II.

AVERAGE GRADIENT FOR ALL METHODS

\begin{tabular}{llll}
\hline & Band 1 & Band 2 & Band 4 \\
\hline Multi-spectral image & 4.5302 & 3.6742 & 4.1685 \\
BT & 16.552 & 15.417 & 15.970 \\
IHS & 11.306 & 10.170 & 10.416 \\
PCA & 13.704 & 13.771 & 13.093 \\
HPF & 10.382 & 10.352 & 10.050 \\
MGS & 14.594 & 14.607 & 14.836 \\
\hline
\end{tabular}

TABLE III.

CORRELATION COEFFICIENT FOR ALL FUSED IMAGES AND SOURCE MULTI-SPCTRAL ONE

\begin{tabular}{llll}
\hline & Band 1 & Band 2 & Band 4 \\
\hline BT & 0.9904 & 0.9702 & 0.9433 \\
IHS & 0.9238 & 0.9482 & 0.9376 \\
PCA & 0.8887 & 0.9324 & 0.8031 \\
HPF & 0.8976 & 0.9066 & 0.9090 \\
MGS & 0.9556 & 0.9554 & 0.9477 \\
\hline
\end{tabular}


Figure 5(a)-(f) show the histograms of the fused images and the source multi-spectral image, from which we can see that the histograms of BT Figure 5(b), HPF Figure 5(e) and MGS Figure 5(f) are similar to that of the source multi-spectral image, especially the MGS method because the shapes of Figure 5(a) and Figure 5(f) are nearly the same. As for BT and HPF, band 2 is slightly different. And IHS Figure 5(c) and PCA Figure 5(d) enjoy entirely different histograms from that of Figure 5(a).

So we can have the following conclusion according to both the above subjective and objective analysis on the fused images produced by varied fusing methods. All the fusing methods can generate an image that integrating spectral and spatial information from source images and the proposed MGS method is superior to other approaches in aspects of clarity, color fidelity and contrast. Besides, several of above mentioned objective indices prove that MGS method can better generate the ideal object image as a result, it is appropriate for investigation of geological disasters.

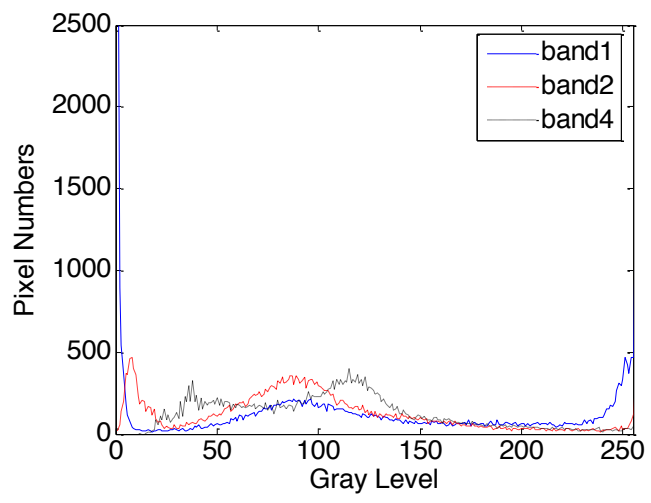

(a)

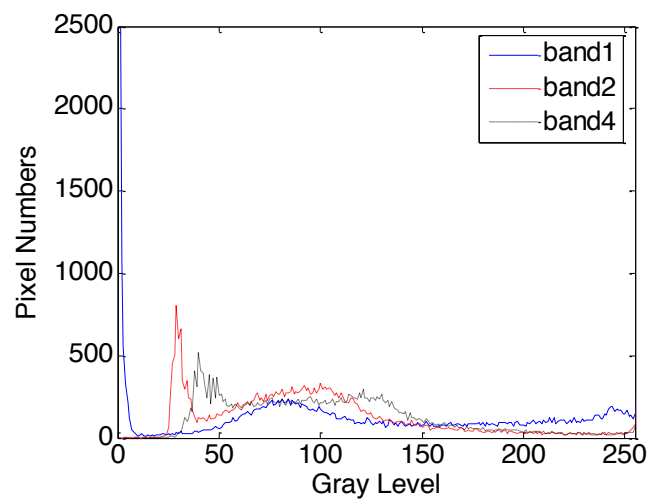

(b)

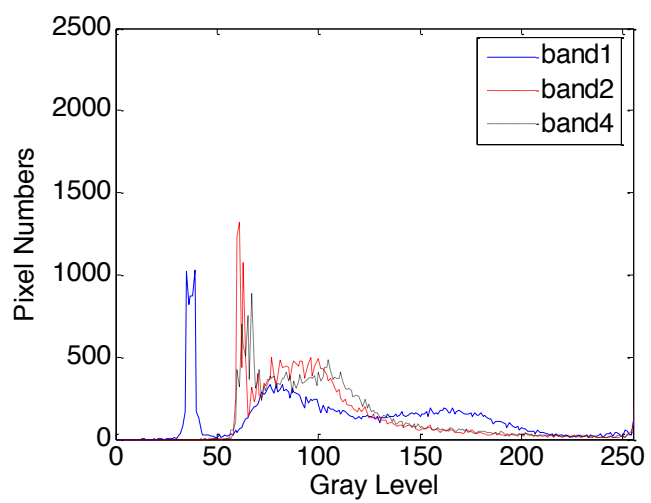

(c)

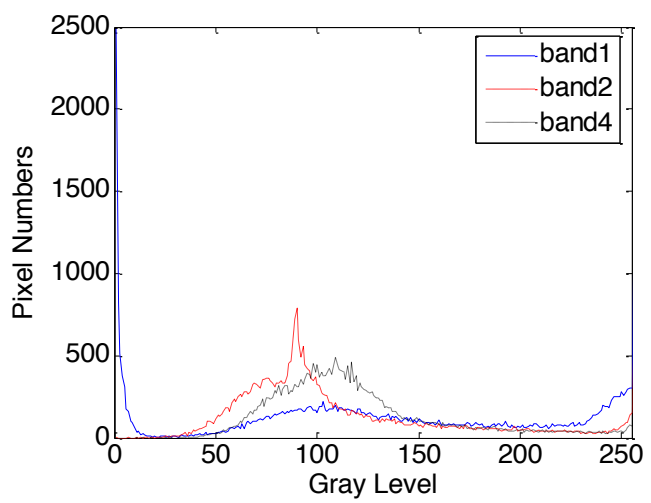

(d)

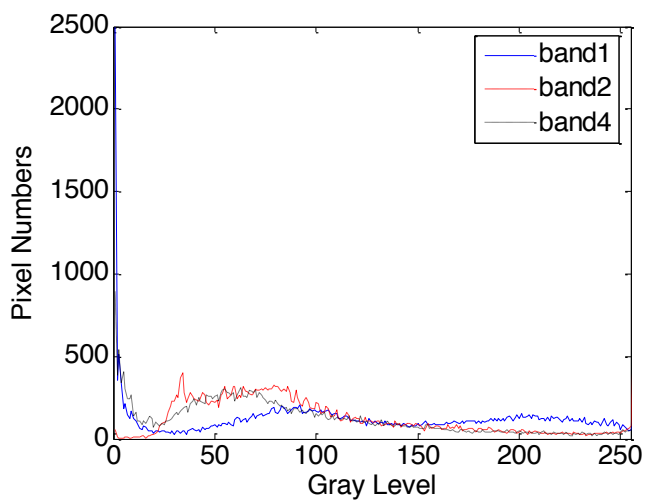

(e)

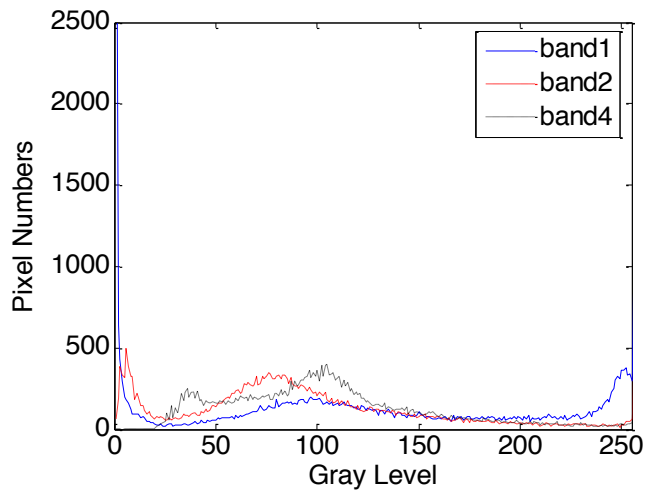

(f)

Figure 5. The histograms of the fused images and the source multispectral image. (a) The source multi-spectral image; (b) BT fused image; (c) IHS fused image; (d) PCA fused image; (e) HPF fused image; (f) MGS fused image.

\section{Evaluation of Geological Disaster INTERPRETATION}

Remote sensing interpretation of geological disasters is featured by characteristics of professionality, pertinence and empiricism. But on the evidence so far, humancomputer interactive interpretation is still the primary means of image quality evaluation and identification of geological disasters. According to the past investigation results of geological hazards and the field survey results, the remote sensing interpretation signs of study area geological disasters are summarized as shown in Table IV. 
TABLE IV

REMOTE SENSING INTERPRETATION OF GEOLOGICAL DISASTERS IN FIELD AREA

\begin{tabular}{ll}
\hline Disaster & Interpretation \\
\hline Landslide has obvious morphological signs and \\
structural characteristics, steep chair-shaped back \\
wall and micro-geomorphologic features are clearly \\
observed. And there are significant differences from \\
the surrounding geological bodies in tone, texture \\
and vegetation growth conditions. \\
Collapse enjoys clear geomorphologic features, \\
which developed in the valley, cliffs on both sides \\
of the river or fracture rock zone. The back wall is \\
usually steep and rough, and stacks to cone at the \\
foot of the slope with lighter hue than surroundings. \\
The contour of the post edge is apparent, the color \\
of the collapse wall is related to lithology and is \\
often with no or sparse vegetation. The collapse is \\
generally straight in longitudinal surface. \\
$\begin{array}{l}\text { Gully debris flow can generally be divided into } \\
\text { three distinct parts: the source area, circulation area } \\
\text { and stacking area, the last one is primarily the } \\
\text { interpretation object. The intuitive method is } \\
\text { usually adopted based on geomorphological type of } \\
\text { the entrance of the gully. If the debris flow fan can } \\
\text { be identified in the image, then label it as debris } \\
\text { flow. The stacking area locates in the outlet of the } \\
\text { valley with gentle slope and often forms alluvial fan } \\
\text { or stacking cone with shallow color, and is } \\
\text { overflowed mostly. }\end{array}$ \\
\hline Debris flow
\end{tabular}

\section{CONCLUSION AND DISCUSSION}

The acquisition of ideal satellite remote sensing data is subject to the passing time and weather conditions, it is impossible to get appropriate remote sensing data under any circumstances, especially for remote sensing survey of geological disasters. In this paper, we have discussed five methods of remote sensing image fusing for investigation of regional geological surveys. From subjective survey and objective analysis we find that different method has different feature, but in most cases, the proposed MGS method can produce better results no matter in visual survey or quantitative analysis.

When conducting geological surveys, remote sensing images need to contain both high spatial resolution and high spectral resolution. Considering the large difference of resolution between multi-spectral ETM+ and SPOT 5 (greater than four times of spatial resolution empirically), so it is uncertain how the fusion effect would be. As the ETM+ image can only provide spatial resolution of $15 \mathrm{~m}$, when an accurate image on geological disasters is needed, a $120 \mathrm{~m}$ long collapse is display on an image only with 8 pixels, which is difficult to make precise interpretation. And the SPOT 5 pan enjoys $2.5 \mathrm{~m}$ spatial resolution, so the fused image is clearer and can provide more pixels on image which is conducive to investigator to make accurate and quick decision.

The experimental results show that all methods adopted in our article can fuse source images effectively, but the proposed MGS method can achieve better result both in subjective and objective evaluations. We choose the MGS in geological image fusion which can meet the interpretation accuracy of geological disaster survey.

\section{REFERENCES}

[1] X. Yao, F.C. Dai, and J. Chen, "Landslide interpretation of remote sensing images in the dry-hot valley of Jinsha River," Chinese. J. Geolog. Hazard. Control, vol. 17, pp. 18-21, 2006.

[2] S. Wan, and Y.Z. Fan, "Design of a remote control vehicle based wireless communication used for fighting fire," Int. J. Online. Eng., vol. 9, pp. 79-82, 2013. http://dx.doi.org/10.3991/ ijoe.v9iS6.2891

[3] D.J. Xun, D.H. Zhang, and H.Z. Wei, "Application of multisource remote sensing image fusion in geohazard investigation," Remote Sensing Tech. Appli., vol. 26, pp. 664-669, 2011.

[4] J.C. Yang, J. Xu, and Z.X. Zhang, "Comparison of SAR and TM images fusion based on PCA transformation with different component," Remote Sensing for Land \& Resources, vol. 66, pp. 73-79, 2000.

[5] Y.H. Jia, D.R. Li, and J.L. Liu, "Comparison of SAR and TM images fusion based on four kinds of IHS transform," J. Rem. S., vol. 13, pp. 103-106, 1998.

[6] C.W. Li, B. Cheng, and Y. Zhang, "A remote sensing image fusion method based on PCA transform and wavelet packet transform," IEEE Int. Conf. Neural Networks \& Signal Processing, vol. 1, pp. 14-17, 2003.

[7] H. Yéson, Y. Besnus, and J.Polet, Zhang, "Extraction of spectral information from Landsat TM data and merger with SPOT panchromatic imagery -- a contribution to be study of geological structures," ISPRS J. Photogrammetry and Rem. S., vol. 48, pp. 23-36, 1998.

[8] H.Y. Yu, B.K. Yan, and F.P. Gan, "Hyper-spectral image fusion by an enhanced Gram Schmidt spectral transformation," Geography and Geo-Information Sci., vol. 23, pp. 39-42, 2007.

[9] J.L. Rodgers, and W.A. Nicewander, "Thirteen ways to look at the correlation coefficient," The American Statistician, vol. 42, pp. 59-66, 1988. http://dx.doi.org/10.2307/2685263

[10] Y.S. Zhou, G. Zhang, and J. Li, "Application of digital image processing technology in interferometer," Int. J. Online. Eng., vol. 9, pp. 65-68, 2013.

[11] J.M. Zheng, M.J. He, and E.D. Liu, etc., "Adaptive fuzzy PID control for servo motor direct-drive pump control system," $J$. Digital Info. Mange., vol. 12, pp. 1-7, 2014.

[12] W.J. Carper, "The use of intensity-hue-saturation transformations for merging SPOT panchromatic and multi-spectral image data," Photogrammetric Eng. and Rem. S., vol. 56, pp. 459-467, 1990.

[13] K. Edwards, and P.A. Davis, "The use of intensity-hue-saturation transformation for producing color shaded-relief images," Photogrammetric Eng. Rem. S., vol. 60, pp. 1369-1374, 1994.

[14] C. Pohl, "Multi-sensor image fusion in remote sensing: concepts, methods and applications," Int. J. Rem. S., vol. 19, pp. 823-854, 1998. http://dx.doi.org/10.1080/014311698215748

[15] P.S. Chaves, and S.C. Sides, "Comparison of three different methods to merge multi-resolution and multi-spectra data:TM \& SPOT pan," Photogrammetric Eng. Rem. S., vol. 57, pp. 295-303, 1991.

\section{AUTHORS}

Juan Wang, she received the BS and MS degrees of computer science from China West Normal University, China in 2003 and 2006 respectively, and now is a Ph.D candidate in Chengdu University of Technology. Her research interests include image processing, pattern recognition and computing intelligence etc. (e-mail: wjuan@126.com).

Huajun Wang, he is a professor and Ph.D supervisor of geophysical institute in Chengdu University of Technology. His published papers have been indexed by SCI or EI. His research interest includes solar wind, information system and computing intelligence etc. (email: hjwang@cdut.edu.cn).

This work was supported in part by a grant from 14ZA0124 of Sichuan provincial education department and $13 \mathrm{C} 003$ of China West Normal University. Submitted 25 March 2014. Published as resubmitted by the authors 08 June 2014 . 